\title{
Prevention of mother-to-child transmission of hepatitis B virus: a phase III, placebo- controlled, double-blind, randomized clinical trial to assess the efficacy and safety of a short course of tenofovir disoproxil fumarate in women with hepatitis $B$ virus e-antigen
}

Gonzague Jourdain ${ }^{1,2,3^{*}}$ (D), Nicole Ngo-Giang-Huong ${ }^{1,2,3}$, Tim R. Cressey ${ }^{1,2,3,4}$, Lei Hua ${ }^{5}$, Linda Harrison ${ }^{5}$, Camlin Tierney ${ }^{5}$, Nicolas Salvadori ${ }^{1,2}$, Luc Decker ${ }^{1,2}$, Patrinee Traisathit ${ }^{14}$, Wasna Sirirungsi ${ }^{2}$,

Woottichai Khamduang ${ }^{2}$, Chureeratana Bowonwatanuwong ${ }^{6}$, Thanyawee Puthanakit ${ }^{7}$, George K. Siberry ${ }^{8}$, Diane Heather Watts ${ }^{9}$, Trudy V. Murphy ${ }^{10}$, Jullapong Achalapong ${ }^{11}$, Suchat Hongsiriwon ${ }^{12}$, Virat Klinbuayaem ${ }^{13}$, Satawat Thongsawat ${ }^{15}$, Raymond T. Chung ${ }^{16}$, Stanislas Pol ${ }^{17}$ and Nantasak Chotivanich ${ }^{6}$

\begin{abstract}
Background: Chronic hepatitis B virus (HBV) infection is complicated by cirrhosis and liver cancer. In Thailand, 6-7 \% of adults are chronically infected with HBV. The risk of mother-to-child transmission (MTCT) of HBV has been estimated to be about $12 \%$ when mothers have a high hepatitis B viral load, even if infants receive passive-active prophylaxis with HBV immunoglobulin ( $\mathrm{HBlg}$ ) and initiate the hepatitis B vaccine series at birth. We designed a study to assess the efficacy and safety of a short course of maternal tenofovir disoproxil fumarate (TDF) among women with a marker of high viral load for the prevention of MTCT of HBV.

Methods: The study is a phase III, multicenter (17 sites in Thailand), placebo-controlled, double-blind, randomized 1:1, two-arm clinical trial of TDF $300 \mathrm{mg}$ once daily versus placebo among pregnant women from 28 weeks' gestation through 2-month post-partum. All infants receive HBlg at birth, and a hepatitis B (HB) vaccination series according to Thai guidelines: birth, and age 1, 2, 4 and 6 months. Participant women at study entry must be age $\geq 18$ years, hepatitis B surface antigen ( $\mathrm{HBsAg}$ ) and e-antigen ( $\mathrm{HBeAg}$ ) positive, have alanine aminotransferase (ALT) level $<30 \mathrm{IU} / \mathrm{L}$ at screening (confirmed $<60 \mathrm{IU} / \mathrm{L}$ pre-entry), negative hepatitis $C$ serology, creatinine clearance $>50 \mathrm{~mL} / \mathrm{min}$, and no history of anti-HBV antiviral treatment.

The target sample size of 328 mother/infant pairs assumed 156 evaluable cases per arm to detect a $\geq 9 \%$ difference in (Continued on next page)
\end{abstract}

\footnotetext{
* Correspondence: gonzague.jourdain@ird.fr

'Institut de recherche pour le développement (IRD, France), UMI 174 - PHPT,

187/10, Changklan Rd., Changklan, Muang, Chiang Mai 50100, Thailand

${ }^{2}$ Chiang Mai University, Faculty of Associated Medical Sciences, 110

Intawaroroj Rd., Sripoom, Chiang Mai 50200, Thailand

Full list of author information is available at the end of the article
} 


\begin{abstract}
(Continued from previous page)
MTCT transmission (3 \% experimental arm versus $12 \%$ placebo arm) with $90 \%$ power. Mothers and infants are

followed until 12 months after delivery. The primary infant endpoint is detection of HBsAg, confirmed by detection

of HBV DNA at six months of age. Secondary endpoints are maternal and infant adverse events, acute exacerbations

of maternal hepatitis B disease (ALT >300 IU/L, defined as a "flare") following discontinuation of study treatment, infant HBV infection status and growth up to 12 months of age.

Discussion: The results of this randomized trial will clarify the efficacy and safety of a short course of antiviral treatment to prevent mother-to-child transmission of HBV and inform international guidelines.
\end{abstract}

Trial registration: ClinicalTrials.gov Identifier NCT01745822.

Keywords: Hepatitis B, Hepatitis B surface antigen, Hepatitis B e antigen, Pregnancy, Mother-to-child transmission, Thailand

\section{Background}

Chronic hepatitis B virus (HBV) infection affects an estimated 240 million persons worldwide, is complicated by cirrhosis of the liver and hepatocellular carcinoma, and causes 650,000 deaths annually [1]. HBV is most infectious in persons with high-level viremia (HBV load) or positive $\mathrm{HBeAg}$, a marker of high-level viral replication. In countries with a high prevalence $(>8 \%)$ of HBsAgpositive persons, and before the implementation of universal immunization programs, most HBV infections were the result of early childhood household transmission or maternal-to-child transmission (MTCT). Compared to other age groups, infants have the highest rate (about $90 \%$ ) of HBV infection progressing to chronic HBV infection. MTCT is thought to occur primarily during birth/delivery, but the timing and predominant route of transmission is poorly understood.

Efficient $\mathrm{HB}$ vaccines have been available since the early 1980s. The World Health Organization (WHO) recommends universal infant immunization, regardless of maternal HBV infection status, starting at birth. This intervention has dramatically reduced the prevalence of HBV infection in children wherever it has been implemented. For infants born to HBV-infected mothers, concurrent administration of $\mathrm{HB}$ immunoglobulin (HBIg) to the neonate as soon as possible after birth for added passive prophylaxis incrementally reduces the risk of infection [2]. However, studies have reported that $8 \%$ to $12 \%$ of infants born to mothers with high-level viremia are infected despite prophylaxis with passive (HBIg) and active (HB vaccine) immunization starting at birth [3-5]. It is possible that in utero transmissions cannot be prevented by interventions initiated after birth. Additionally, HBV escape mutants to HB vaccine- or HBIg- may be responsible for some transmissions [6, 7].

Studies have suggested that antivirals inhibiting HBV replication such as lamivudine, tenofovir disoproxil fumarate (TDF), and telbivudine, prescribed to pregnant women with high HBV loads at the end of pregnancy and during the early postpartum period can reduce the risk of MTCT [3, 4, 8]. This scientific evidence has been weakened by methodological shortcomings in the studies conducted to date. A recent meta-analysis and review concluded that "most of the data [were] derived from cohort studies, which are subject to significant biases, especially selection bias," and stressed the "absence of studies warranting high confidence" and "a paucity of randomized clinical trials" [9]. This led the American Association for the Study of Liver Diseases not to recommend but rather "suggest antiviral therapy to reduce the risk of perinatal transmission of hepatitis $B$ in HBsAg-positive pregnant women with an HBV DNA level $>200,000 \mathrm{IU} / \mathrm{mL}$ ", rating the Quality/Certainty of Evidence as "Low" and the Strength of Recommendation as "Conditional" [10]. Similarly, in 2015, the first WHO Hepatitis Guidelines did not recommend this approach for the sole purpose of preventing MTCT of HBV (quality of evidence rated as "Very low"), even though some "countries in Asia have adopted a policy of treating highly viremic pregnant mothers with lamivudine, telbivudine or tenofovir" [1]. Moreover, the safety of the approach has not been fully assessed, specifically the risk of hepatic disease exacerbation (flare) following postpartum discontinuation of antiviral treatment.

The protocol described here was designed to address essential questions about the efficacy and safety of providing antiviral therapy to pregnant women who have markers of high HBV load to prevent MTCT of HBV. Indeed recent guidelines and reviews still stress the need for well conducted randomized clinical trials with sufficient sample size $[1,9,10]$. This study is being conducted in Thailand where antiviral treatment of pregnant women has not been implemented.

We hypothesize that a short course of TDF compared to placebo will reduce the risk of MTCT of HBV among HBeAg positive women, --likely through a decrease in HBV load and, possibly, through a prophylactic effect in the fetus in combination with passive-active prophylaxis at birth in the neonate-, and that only clinically 
insignificant maternal flares will occur with postpartum antiviral discontinuation.

\section{Methods}

This is a phase III, multicenter, placebo-controlled, double-blind, randomized (1:1), two parallel arm clinical trial conducted in 17 public hospitals in Thailand (see Fig. 1). The list of locations is available at ClinicalTrials.gov Identifier NCT01745822. The primary objective is to assess the efficacy of TDF for the prevention of MTCT of HBV at 6 months of age. Important secondary objectives are to assess the risk of postpartum hepatic disease exacerbation after antiviral discontinuation, and infant growth through 12 months of age as part of the safety assessments.

Pregnant women are tested for the presence of HBsAg at their first antenatal care visit as standard of care in Thailand. At each site, all pregnant women aged 18 years or older with a positive HBsAg test were informed about the study and invited to provide their informed consent for participation.

The following study eligibility criteria are verified at enrollment:

Inclusion Criteria:

- Pregnancy

- At least 18 years of age

- Negative Human Immunodeficiency Virus serology

- Positive HBsAg and HBeAg tests

- Gestational age of 28 weeks (+ or - 10 days) as determined by the study site obstetrician

- Alanine aminotransferase (ALT) $\leq 30 \mathrm{IU} / \mathrm{L}$, confirmed $\leq 60 \mathrm{IU} / \mathrm{L}$ on a subsequent blood draw

- Pregnant woman agrees to bring her infant at the planned study visits at a study site, until one year after delivery and to inform the site investigator if she plans to move to another place and not be able to return to the clinic.

- Pregnant woman understands the need for adequate infant immunization and agrees to the blood draws from her infant and the need for close follow-up to manage possible exacerbation of hepatitis.

\section{Exclusion Criteria:}

- History of TDF treatment at any time, or any other anti-HBV treatment during the current pregnancy.

- Creatinine clearance $<50 \mathrm{~mL} / \mathrm{min}$, calculated using the Cockcroft-Gault formula.

- Dipstick proteinuria $>1+(>30 \mathrm{mg} / \mathrm{dL})$ or normoglycemic glucosuria confirmed on two separate occasions.

- Positive serology for Hepatitis C infection.

- Evidence of fetal anomalies incompatible with life.

- Any concomitant condition or treatment that, in the view of the clinical site investigator, would contraindicate participation or satisfactory follow-up in the study.

- Concurrent participation in any other clinical trial without written agreement of the two study teams.

At 28 weeks gestation (+/-10 days), eligible participants are enrolled and randomly assigned to receive the study treatment (manufactured by Gilead Sciences Inc.) of either TDF $300 \mathrm{mg}$ once daily in the "TDF" experimental arm or a matching placebo in the reference arm, from 28 weeks gestation through 2-months postpartum. Women are instructed to return to the study pharmacist any unused study treatment and empty bottles;

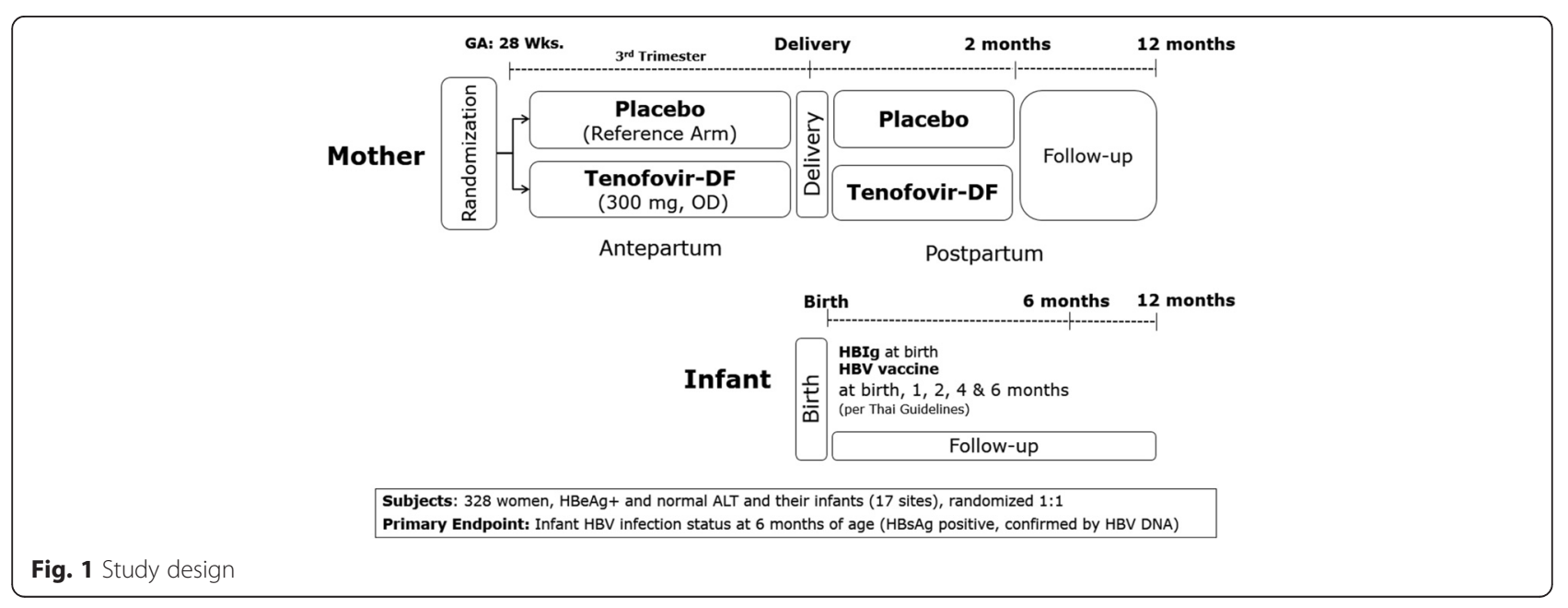


adherence to treatment is assessed by self-report and exact pill count. All infants receive passive-active prophylaxis with HBIg at birth and $\mathrm{HB}$ vaccination according to Thai guidelines: at birth, and ages 1, 2, 4 and 6 months of age. Following WHO guidelines, mothers are encouraged to breastfeed as there is no evidence of additional transmission during breastfeeding $[11,12]$.

Mothers and infants are followed until 12 months after delivery. Maternal study visits are conducted at 28, 32, 36 weeks' gestation, delivery, and at 1, 2, 3, 4, 6 and 12 months postpartum. For mothers with confirmed moderate liver disease exacerbation (ALT >60 IU/L) after discontinuation of the study treatment, specific evaluations are conducted including bilirubin, prothrombin/International Normalized Ratio, and albumin, and plasma is stored for further retrospective evaluations. Additional investigations are performed for case management and documentation, and reintroduction of the double-blind study treatment is considered following a pre-defined algorithm by the reference site internist if high ALT levels persist. Infant visits are scheduled at birth, 1, 2, 4, 6, 9 and 12 months of age for clinical safety evaluation and determination of HBV status.

Serious adverse events (SAEs) that occur in either arm, as defined by 1996 International Conference on Harmonisation Good Clinical Practice, whether or not considered to be related to the study treatment by the study site investigator, are immediately reported to the Thai Ministry of Public Health and to the Eunice Kennedy Shriver National Institute of Child Health \& Human Development (NICHD) and Centers for Disease Control and Prevention (CDC) program scientists, and those related to the study treatment to the drug manufacturer.

The primary endpoint is the detection of HBsAg that is confirmed by detectable HBV DNA in infants at six months of age. Secondary endpoints are infant's HBV infection status, defined as detection of HBsAg confirmed by detectable HBV DNA at or after 6 months through 12 months of age, occurrence of maternal and infant adverse events (SAEs and grade 3/4 signs and symptoms in the Division of AIDS Table for Grading the Severity of Adult and Pediatric Adverse Events, Version 1.0, December, 2004 [13]), occurrence of flares (ALT >300 IU/L) following discontinuation of study treatment, and infant growth measured by weight, height and head-circumference at 6 and 12 months of age.

\section{Randomization, allocation concealment, and blinding}

The sequentially numbered permuted blocks (of size undisclosed to the investigators) randomization list was computer generated by the study statistician (who had no further involvement in study treatment allocation) before the study started. The list, securely transmitted to the study pharmacist only, is used for secondary packaging of study treatment, consisting of 30-tablet bottles, into sequentially ordered packages. Stratification by site is achieved by shipping packages of study treatment divisible by the block size and blinding is achieved by only identifying study treatment by the patient identification number dictated by the sequential list. Patients, study staff on site and at the coordination center, investigators and laboratory personnel in charge of $\mathrm{HBV}$ related tests are blind to the randomized assignment.

\section{Sample size}

It was calculated that a total of 312 evaluable motherinfant pairs were needed to detect a $\geq 9 \%$ difference in HBV transmission between arms (3\% in the experimental arm versus $12 \%$ in the placebo arm) with $90 \%$ power using a Fisher's exact test and a one-sided 0.049 significance level to account for one interim efficacy analysis. This difference is equivalent to a relative reduction of $75 \%$ in $\mathrm{HBV}$ infection rate in the TDF arm. It was planned to enroll 328 pregnant women to account for an estimated $5 \%$ loss to follow-up. If the infection rate in the placebo arm is only $10 \%$, the study has $83.2 \%$ power to detect a $75 \%$ reduction in the infection rate, i.e., an infection rate at $2.5 \%$ in the TDF arm. However, if the infection rate is only $8 \%$, the power of detecting a $75 \%$ reduction in the infection rate $(2 \%$ infection rate in the placebo arm) drops to $72.6 \%$.

\section{Monitoring}

The composition of the Data and Safety Monitoring Board (DSMB) adhered to the following criteria: at least 1 gastroenterologist/hepatologist, 1 pediatrician, 1 obstetrician, 1 infectious diseases specialist, and 1 statistician/ epidemiologist, at least 3 of them from Thailand. Program scientists participate as observers in open sessions of DSMB calls/meetings. The study was presented to the DSMB during a protocol initiation review before study initiation. At annual meetings, the DSMB reviews the conduct of the study and adverse events. One interim review to monitor efficacy is planned when $50 \%$ of the information on the primary endpoint is available, i.e. when 164 infants either have their 6-month HBV status available or are off study before the 6-month visit. A Haybittle-Peto function is employed for the group sequential design, so that at the interim efficacy review, the comparison of the primary endpoint is considered significant only if $p<0.001$.

\section{Statistical analyses}

For the primary objective/endpoint, the proportions of infants with positive HBsAg, confirmed by HBV DNA, at 6 months of age, along with their $95 \%$ confidence intervals, will be provided by arm based on the exact 
Binomial distribution and compared between the TDF arm and the placebo arm based on Fisher's exact test. The test will be adjusted for the interim efficacy monitoring by considering $p<0.05-0.001=0.049$ as significant. If mothers' baseline HBV DNA loads are imbalanced between treatment arms, an exact logistic regression will be conducted as a sensitivity analysis. The primary analysis will be conducted as a complete case analysis including infants whose 6-month endpoint is available in their original randomized group, regardless of the duration of study treatment their mothers actually received - if at least one dose was taken. A secondary analysis will be conducted in a modified intent to treat (mITT) population, where all randomized pregnant women who received at least one dose of study treatment will be included, and infants who are lost to follow-up before their 6 months HBV status is available will be considered as infected. Further sensitivity analysis may be performed, including imputing infants' last available HBV infection status as their 6 months status for those who are lost to follow-up prior to 6 months or multiple imputations accounting for possible reasons of loss to follow-up, to explore the effect of loss to follow-up. A secondary supporting analysis will be conducted also considering as $\mathrm{HBV}$ infected infants with positive HBsAg, confirmed by HBV DNA, after 6 months and up to 12 months of age. Logistic regression, accounting for factors that are known to be associated with mother-to-child HBV transmission, could also be conducted to provide an adjusted odds ratio between treatment arms.

For secondary objectives/endpoints, proportions of women with severe hepatic disease exacerbation (ALT $>300 \mathrm{IU} / \mathrm{L}$, regardless of baseline values) occurring after study treatment discontinuation and up to 12 months postpartum will be compared between treatment arms by Fisher's exact test. Proportions of women with any adverse event (any SAE or grade 3/4 sign or symptom defined by the Division of AIDS) from randomization until study exit will be analyzed similarly. Poisson regressions, with an offset of follow-up time will be further conducted to model repeated occurrence of adverse events. Adverse events in infants will be analyzed in a similar manner. Infants' mean weight, height and head circumference z-scores at 6 and 12 months of age will be compared between arms based on normal approximation and two-sample t-tests.

In exploratory analysis, the proportion of infants with detectable 9 month HBV DNA and that of infants with seroprotection (anti-HBs antibodies $>10 \mathrm{IU} / \mathrm{L}$ ) will be compared by treatment arm, as well as the proportions of premature labors (birth before 37 weeks of gestational age assessed by Ballard Score). Additional exploratory analyses include, but are not limited to, analysis on time to treatment discontinuation and/or study discontinuation and analysis on adherence.

Quarterly conference calls involving the core protocol team and program scientists are held to review the progress of the study and adverse events. The implementation of the study at each site is audited by a contract research organization one to three times during the study period, depending on the number of enrollees.

All site investigators have been trained in Good Clinical Practice and Human Subjects Protection. The trial protocol was reviewed and approved on September 3, 2012 by the Ethics Committees of the Institute for the Development of Human Research Protections at the Thailand's Ministry of Public Health (reference สคม 1340/ 2555), the Faculty of Associated Medical Sciences, Chiang Mai University, on October 18, 2012 (reference $525 / 2555$ ), the NICHD, and the CDC, as well as by the ethics committees of participating sites in Thailand.

\section{Discussion}

This phase III randomized placebo-controlled doubleblind trial will assess the efficacy and safety of a short course antiviral treatment to prevent mother-to-child transmission of HBV. Enrollment was opened in January 2013 and accrual was completed on August 19, 2015 with no significant operational issues. The data collection for the primary endpoint is scheduled to be completed in August 2016. Primary efficacy results are expected soon afterwards. The efficacy and safety study results will help clarify whether this strategy can play a significant role in the prevention of mother-to-child HBV transmission in highly viremic women.

\section{Abbreviations}

ALT, alanine aminotransferase; CDC, centers for disease control and prevention; DSMB, data and safety monitoring board; $\mathrm{HB}$, hepatitis $\mathrm{B}$; $\mathrm{HBeAg}$ : hepatitis B e-antigen; HBlg, hepatitis B specific immunoglobulin; HBsAg, hepatitis B surface antigen; HBV, hepatitis B virus; IU, international units; mITT, modified intent to treat; MTCT, mother-to-child transmission; NICHD, Eunice Kennedy Shriver National Institute of Child Health \& Human Development; SAE, Serious adverse events; TDF, tenofovir disoproxil fumarate; WHO, World Health Organization

\section{Acknowledgements \\ We are grateful to Amy Sandul, Centers for Disease Control and Prevention, for careful and helpful editing of the manuscript. \\ Funding \\ The study activities are supported by a grant from the Eunice Kennedy Shriver National Institute of Child Health \& Human Development (NICHD) (U01HD071889) under a cooperative agreement between NICHD, the Centers for Disease Control and Prevention, United States of America, and Institut de recherche pour le développement, France. \\ Study drugs (tenofovir disoproxil fumarate and matching placebo) were donated by Gilead Sciences Inc., CA, United States of America.}

Availability of data and materials Not applicable. 


\section{Authors' contributions}

GJ and SP conceived the idea of the study. GJ developed the study and its design, wrote the first draft and revised the manuscript. NNGH, TRC made important contributions to the design of the study. LHu, LHa, CT, NS and LD contributed to the statistical analysis plan. All authors participated in the design of the study, reviewed the manuscript and approved the final manuscript (DHW contributed to the study when working for the Eunice Kennedy Shriver National Institute of Child Health and Human Development).

\section{Competing interests}

The authors declare that they have no competing interests.

\section{Consent for publication}

not applicable.

\section{Ethics approval and consent to participate}

Institute for the Development of Human Research Protections (IHRP) at the Ministry of Public Health of Thailand; Ethics committee of the Faculty of Associated Medical Sciences, Chiang Mai University; local ethics committees of the following provincial and regional hospitals: Chiangrai Prachanukroh, Chonburi, Prapokklao, Samutsakhon, Khon Kaen, Nopparat Rajathanee, Maharat Nakhon Ratchasima, and the Bhumibol Adulyadej Hospital (Royal Air Force). Public hospitals with no ethics committees recognized the review by the Ministry of Public Health ethics committee.

\section{Author details}

'Institut de recherche pour le développement (IRD, France), UMI 174 - PHPT, 187/10, Changklan Rd., Changklan, Muang, Chiang Mai 50100, Thailand. ${ }^{2}$ Chiang Mai University, Faculty of Associated Medical Sciences, 110 Intawaroroj Rd., Sripoom, Chiang Mai 50200, Thailand. ${ }^{3}$ Department of Immunology and Infectious Diseases, Harvard T.H. Chan School of Public Health, 677 Huntington Ave, Boston, MA 02115, USA. ${ }^{4}$ Department of Molecular \& Clinical Pharmacology, Institute of Translational Medicine, University of Liverpool, Liverpool, UK. ${ }^{5}$ Center for Biostatistics in AIDS Research (CBAR), Harvard T.H. Chan School of Public Health, 677 Huntington Ave, Boston, MA 02115, USA. ${ }^{6}$ Chonburi Hospital, 69 M.2, Sukhumvit Rd., Ban-suan, Muang, Chonburi 20000, Thailand. ${ }^{7}$ Chulalongkorn University, Faculty of Medicine, 1873 Rama 4 Road, Pathumwan, Bangkok 10330, Thailand. ${ }^{8}$ Eunice Kennedy Shriver National Institute of Child Health and Human Development, National Institutes of Health, 6100 Executive Blvd, Bethesda, MD 20892, USA. ' US Department of State, Office of the Global AIDS Coordinator, SA-22, 2201 C Street NW, Washington, DC 20522-2210, USA. ${ }^{10}$ Centers for Disease Control and Prevention, DHHS/CDC//NCHHSTP/ DVH/Naccine Unit Bldg Corporate SQ 12, Room 3111, Atlanta, GA 30329-1902, USA. ${ }^{11}$ Chiangrai Prachanukroh Hospital, Obstetrics \& Gynecology Department, 1039 Sathan Phayaban Rd., Muang, Chiang Rai 57000, Thailand. ${ }^{12}$ Chonburi Hospital, Pediatrics Department, 69 M.2, Sukhumvit Rd., Ban-suan, Muang, Chonburi 20000, Thailand. ${ }^{13}$ Sanpatong Hospital, Medical Department, 149 M.15 Yuhwa, Sanpatong, Chiang Mai 50120, Thailand. ${ }^{14}$ Department of Statistics, Chiang Mai University, Faculty of Science, 239 Huaykaew Rd., Suthep, Muang, Chiang Mai 50200, Thailand. ${ }^{15}$ Department of Internal Medicine, Chiang Mai University, Faculty of Medicine, Muang, Chiang Mai 50200, Thailand. ${ }^{16}$ Massachusetts General Hospital, Gastrointestinal Unit, WRN 1007C, GI Unit, 55 Fruit St, Boston, MA 02114, USA. ${ }^{17}$ Department of Hepato-Gastroenterology, Cochin University Hospital, 27 rue du Faubourg Saint-Jacques, 75679 Paris, Cedex 14, France.

Received: 13 June 2016 Accepted: 25 July 2016

Published online: 09 August 2016

\section{References}

1. WHO. Prevention, care and treatment of persons with chronic hepatitis B infection. Guidelines [Internet]. WHO; 2015 [cited 2016 Feb 29]. Available from: http://apps.who.int/iris/bitstream/10665/154590/1/ 9789241549059 eng.pdf

2. Lee C, Gong Y, Brok J, Boxall EH, Gluud C. Effect of hepatitis B immunisation in newborn infants of mothers positive for hepatitis B surface antigen: systematic review and meta-analysis. BMJ. 2006;332:328-36.

3. Xu W-M, Cui Y-T, Wang L, Yang H, Liang Z-O, Li X-M, et al. Lamivudine in late pregnancy to prevent perinatal transmission of hepatitis $B$ virus infection: a multicentre, randomized, double-blind, placebo-controlled study. J Viral Hepat. 2009:16:94-103.

4. Han G-R, Cao M-K, Zhao W, Jiang H-X, Wang C-M, Bai S-F, et al. A prospective and open-label study for the efficacy and safety of telbivudine in pregnancy for the prevention of perinatal transmission of hepatitis B virus infection. J Hepatol. 2011;55:1215-21.

5. Wen W-H, Chang M-H, Zhao L-L, Ni Y-H, Hsu H-Y, Wu J-F, et al. Mother-toinfant transmission of hepatitis $B$ virus infection: significance of maternal viral load and strategies for intervention. J Hepatol. 2013;59:24-30.

6. Oon CJ, Chen WN. Current aspects of hepatitis B surface antigen mutants in Singapore. J Viral Hepat. 1998:5 Suppl 2:17-23.

7. Carman WF, Zanetti AR, Karayiannis P, Waters J, Manzillo G, Tanzi E, et al. Vaccine-induced escape mutant of hepatitis B virus. Lancet. 1990;336:325-9.

8. Chen H-L, Lee C-N, Chang C-H, Ni Y-H, Shyu M-K, Chen S-M, et al. Efficacy of maternal tenofovir disoproxil fumarate in interrupting mother-to-infant transmission of hepatitis B virus. Hepatology. 2015;62:375-86.

9. Brown RS, McMahon BJ, Lok ASF, Wong JB, Ahmed AT, Mouchli MA, et al. Antiviral therapy in chronic hepatitis B viral infection during pregnancy: a systematic review and meta-analysis. Hepatology. 2016;63:319-33.

10. Terrault NA, Bzowej NH, Chang K-M, Hwang JP, Jonas MM, Murad MH. AASLD guidelines for treatment of chronic hepatitis B. Hepatology. 2016:63:261-83

11. World Health Organization. Hepatitis B and breastfeeding [Internet]. World Health Organization; 1996 [cited 2012 Jul 9]. Available from: http://www. who.int/maternal_child_adolescent/documents/pdfs/hepatitis_b_and_ breastfeeding.pdf

12. Guidelines for the Prevention, Care and Treatment of Persons with Chronic Hepatitis B Infection [Internet]. Geneva: World Health Organization; 2015 [cited 2016 Jun 9]. Available from: http://www.ncbi.nlm.nih.gov/books/ NBK305553/

13. Division of AIDS Table for Grading the Severity of Adult and Pediatric Adverse Events [Internet]. National Institute of Allergy and Infectious Diseases, National Institutes of Health; 2004. Available from: http://rsc.techres.com/document/safetyandpharmacovigilance/table_for_grading_ severity of adult_pediatric adverse events.pdf

\section{Submit your next manuscript to BioMed Central} and we will help you at every step:

- We accept pre-submission inquiries

- Our selector tool helps you to find the most relevant journal

- We provide round the clock customer support

- Convenient online submission

- Thorough peer review

- Inclusion in PubMed and all major indexing services

- Maximum visibility for your research

Submit your manuscript at www.biomedcentral.com/submit
Biomed Central 